\title{
VASCULAR FLORA OF ALABASTER CAVERNS STATE PARK, CIMARRON GYPSUM HILLS, WOODWARD COUNTY, OKLAHOMA
}

\author{
Gloria M. Caddell \\ Department of Biology \\ University of Central Oklahoma \\ Edmond, OK 73034 \\ gcaddell@uco.edu
}

\author{
Kristi D. Rice \\ Department of Biology \\ University of Central Oklahoma \\ Edmond, OK 73034 \\ Current Address: \\ College of Pharmacy \\ University of Oklahoma \\ Oklahoma City, OK 73117
}

Keywords: flora, vascular plants, gypsum, plant distribution

\begin{abstract}
Alabaster Caverns State Park is located in the Cimarron Gypsum Hills of northwestern Oklahoma, a semi-arid region of the state. The majority of the park is dominated by mixed-grass prairie and gypsum outcrops, with some riparian habitat and wooded north-facing slopes. A vascular plant inventory conducted from 2004 through 2007 yielded 274 species in 199 genera and 66 families. The largest families were the Poaceae (52 species), Asteraceae (47), and Fabaceae (23). There were 100 annuals, 6 biennials, and 163 perennials, as well as 5 species that have more than one life history form. Forty-two species $(15.3 \%)$ were not native to North America. Three taxa currently being tracked by the Oklahoma Natural Heritage Inventory (2012) were present: Echinocereus reichenbachii (S3G5), Haploesthes greggii (S1G4?), and Marsilea vestita (S1G5). Compared to floristic inventories of sites in the Cimarron Gypsum Hills that are less impacted by public visitation, but more intensively grazed, Alabaster Caverns State Park has a higher number of species as well as a higher proportion of introduced species.

\section{INTRODUCTION}

Palmer et al. (1995) summarized the importance of floristic inventories in providing data for research on biodiversity, environmental impact assessment, and management decisions. The need for further studies of the vascular flora of the Gypsum Hills Physiographic Province was noted by Hoagland (2000). Since that time, two publications have provided floristic inventories of areas within the Cimarron Gypsum Hills of northwestern Oklahoma. Buckallew and Caddell (2003, 2004) summarized the vascular flora of the Selman Living Laboratory, located approximately 6 miles west of Alabaster Caverns State Park in Woodward County. It supports primarily mixed-grass prairie and gypsum outcrop

communities and was part of the Selman Ranch until 1998. Hoagland and Buthod (2005) surveyed a gypsum-dominated, currently-grazed ranch located approximately 24 miles southeast of Alabaster Caverns in Major County. Alabaster Caverns was established as a state park in the 1950s and therefore has a different land-use history. It is heavily visited by the public and is a site on the Western Oklahoma Wildlife Trail. The objectives of this inventory were to contribute to our knowledge of plant distributions in Oklahoma and in the Cimarron Gypsum Hills; to compare the vascular flora of Alabaster Caverns State Park to that of previously-described, more intensively- grazed but less heavily-visited sites in the Cimarron Gypsum Hills; and to provide a resource that
\end{abstract}

(C) Gloria M. Caddell, Kristi D. Rice

Journal compilation (C) 2012 Oklahoma Native Plant Society 
can be used by state park personnel for education and conservation purposes.

\section{STUDY AREA}

Alabaster Caverns State Park is located in Woodward County, Oklahoma $\left(36^{\circ} 42^{\prime} 00^{\prime}\right.$ N -99 $08^{\prime} 47^{\prime \prime} W ;$; $26 \mathrm{~N}$ R18W SW1/4 of Sec. 28 and NW1/4 of NW1/4 of Sec. 33). The land for the park was purchased by the State of Oklahoma in 1953. It became a state park in 1956 (Allen 2007) and is managed by the Oklahoma Tourism and Recreation Department. The park consists of approximately 81 hectares (=200 acres). Cedar Creek, a tributary of Long Creek, flows west to east through Cedar Canyon and roughly bisects the park. Elevation ranges from about $488 \mathrm{~m}$ to $532 \mathrm{~m}$.

The climate is semi-arid. According to climate data for Woodward County (Oklahoma Climatological Survey 2012), average annual precipitation is about $61 \mathrm{~cm}$. The growing season lasts approximately 186 days, from mid-April to mid-October. The mean annual temperature is $15.6^{\circ} \mathrm{C}$, with daily average temperatures ranging from $2.0^{\circ} \mathrm{C}$ in January to $27.8^{\circ} \mathrm{C}$ in July. Temperatures range from an average low of $-5.6^{\circ} \mathrm{C}$ in January to an average daytime high of $35^{\circ} \mathrm{C}$ in July. Winds average 11 miles per hour and most often are from the south or southwest.

Alabaster Caverns lies in the Cimarron Gypsum Hills Province of Oklahoma (Curtis et al. 1979). Most of the park is underlain by the Blaine Formation, consisting of alternating layers of gypsum and shale formed during the Permian Period. The gypsum outcrops on the site belong to this formation. The Flowerpot Shale, which underlies the Blaine Formation, is exposed in Cedar Canyon (Meyers et al. 1969). Soils belong to the Vernon-

Cottonwood Association and are excessivelydrained loams and clay loams that have formed from gypsum and gypsiferous shales (Nance et al. 1963). The potential vegetation type is mixed grass (Duck and Fletcher 1943).

\section{METHODS}

We intensively surveyed the site throughout the growing seasons of 2004 and 2005. During those years, we visited the site 19 times, from May through October of 2004, and from April to October of 2005. We also surveyed the site in March and May of 2006. During most visits, we walked the areas both north and south of the canyon, and attempted to visit all major habitats within the park. We recorded all vascular plant species we encountered, noted whether they were in flower or fruit, and collected voucher specimens. We collected exotic species only from naturalized populations, excluding cultivated species from around the visitor center and campgrounds. A few species were identified by sight and documented only by photographs, generally because of their rarity at the site or their rarity status in Oklahoma. We added a few species to our vascular plant species list during plot sampling in 2006 and 2007 for a study of the vascular plant communities across the Cimarron Gypsum Hills (Rice 2008). References used for specimen identification included Hitchcock (1971), Great Plains Flora Association (1986), Diggs et al. (1999), Tyrl et al. (2005, 2010), and Barkworth et al. (2007). The organization of taxa in our species list is based on Angiosperm Phylogeny Group (APG III) recommendations (Stevens 2012), and nomenclature follows the PLANTS Database compiled by the United States Department of Agriculture, Natural Resources Conservation Service (USDA, NRCS 2012). The PLANTS Database was also used to determine whether each species was native to North America or introduced, and whether it was an annual, biennial, or perennial. In cases where species have more than one life form across their range, we noted the life form(s) encountered at Alabaster Caverns State Park. Voucher specimens were deposited in the University of Central Oklahoma (CSU) Herbarium.

(C) Gloria M. Caddell, Kristi D. Rice

Journal compilation (C) 2012 Oklahoma Native Plant Society 


\section{RESULTS AND DISCUSSION}

We identified 274 species in 199 genera and 66 families (Table 1, Appendix). These included 4 monilophyes (1 species of horsetail and 3 ferns), 1 gymnosperm, 210 eudicots, and 59 monocots. There was one additional subspecific taxon. Species in the Poaceae (52), Asteraceae (47), and Fabaceae (23) far outnumbered those in other families. Only 7 other families were represented by more than 5 species: Euphorbiaceae (11), Brassicaceae (8), Caryophyllaceae (7), Plantaginaceae (7), Solanaceae (7), Apocynaceae (6), and Onagraceae (6). The largest genera were Astragalus (6 species), Oenothera (6), Chamaesyce (5), and Asclepias (5). One hundred species were annuals, 6 were biennials, and 163 were perennials. Five species had more than one life form. Thirty-six species were trees (18 species), shrubs (12), or woody vines (6). Cylindropuntia imbricata is included on the species list because it apparently has escaped from cultivation within the park.

Three taxa tracked by the Oklahoma Natural Heritage Inventory (2012) were present: Marsilea vestita (S1G5), Haploesthes greggii (S1G4?), and Echinocereus reichenbachii (S3G5). Rarity ranks, in parentheses, range from 1 (critically imperiled) to 5 (demonstrably secure) at the state $(\mathrm{S})$ and global (G) levels.

The park includes primarily mixed-grass prairie and gypsum outcrop plant communities. The major plant association (Hoagland 2000) is the Schizachyrium scopariumCastilleja purpurea var. citrina-Lesquerella gordonii herbaceous association. The north-facing slopes are wooded, and the ravines of Cedar Canyon are dominated by Juniperus virginiana. The areas adjacent to the visitor center and within and adjacent to the park's two campgrounds are disturbed. Although the area south of the canyon has not been grazed since the 1950s, the area north of Cedar Canyon was leased for grazing until 1997 (Caywood 2006), and contains some old-field vegetation. Wetland and riparian vegetation is found along Cedar Creek and on the edges of a pond near the western boundary of the park.

Forty-two species $(15.3 \%)$ in 16 families were not native to North America. Four of these species (Bothriochloa ischaemum, Bromus tectorum, Sorghum halepense, and Tamarix ramosissima) are listed as Oklahoma problem species, 4 (Ailanthus altissima, Erodium cicutarium, Melilotus officinalis, and Ulmus pumila) are on the Oklahoma Watch List, and 14 are problems in border states (Oklahoma Invasive Plants Council 2012). Seventeen species of Poaceae were introduced.

Compared with the recently-grazed Selman Living Lab (Buckallew and Caddell 2003, 2004) and the currently-grazed Major County ranch (Hoagland and Buthod 2005), Alabaster Caverns State Park had a higher number of plant species, although it is smaller (81 ha) than the Selman Living Lab (129.5 ha) and approximately the same size as the Major County ranch $(80+\mathrm{ha})$. The higher number of species is in part due to the higher number and proportion of introduced species at Alabaster Caverns. Of the 229 species at the Selman Living Lab, 21 (9\%) were introduced. Of the 233 species at the Major County ranch, $22(10.6 \%)$ were introduced. The higher number of introduced species at Alabaster Caverns can be attributed to disturbance associated with the high number of visitors to the park, especially around the visitor center and campgrounds. Of the 274 species at Alabaster Caverns State Park, 175 also occur at the Selman Living Lab. Of the 99 species that occur at Alabaster Caverns but not at the Selman Living Lab, 33 are introduced species. Other differences in species composition are due to differences in land-use history and habitats between the two sites; the Selman Living Lab had been recently grazed when it was inventoried, and it includes sandsage grassland as well as a much larger floodplain than Alabaster Caverns State Park. Although the northern part of Alabaster Caverns State Park was grazed recently, the southern part has not been grazed since the 1950s. Because the Selman Living Lab is located only 6 miles

(C) Gloria M. Caddell, Kristi D. Rice

Journal compilation (C) 2012 Oklahoma Native Plant Society 
to the west of Alabaster Caverns State Park, annual temperature and precipitation are the same, and therefore do not contribute to differences in species composition. Alabaster Caverns State Park shares 163 species with the Major County ranch. Differences in species composition between those 2 sites can be attributed in part to their different grazing histories as well as to some differences in habitats. The Major County Ranch is grazed currently, and contains a large pond, disturbed areas associated with oil well pads, and more roads than Alabaster Caverns State Park. Environmental factors also differ between the sites. Although average temperature differs by only $1^{\circ} \mathrm{C}$, average annual precipitation is approximately $61 \mathrm{~cm}$ for Alabaster Caverns State Park and approximately $70 \mathrm{~cm}$ for the Major County ranch.

The major vegetation associations at Alabaster Caverns and brief descriptions of common species are as follows:

\section{Schizachyrium scoparium-Castilleja purpurea var. citrina-Lesquerella gordonii herbaceous association}

This was the predominant vegetation association in the park, on the gypsum outcrops and shallow soils on gypsum (Figures 1-3). Common associated species included Aristida purpurea, Boutelona curtipendula, Boutelona gracilis, Chamaesyce glyptosperma, Croton monanthogynous, Dalea enneandra, Echinocereus reichenbachii, Heterotheca stenophylla, Lithospermum incisum, Mentzelia nuda, Mentrelia oligosperma, Nama stevensii, Oenothera hartwegii, Oenothera serrulata, Opuntia phaeacantha, Paronychia jamesii, Phacelia integrifolia, Polanisia dodecandra, Polygala alba, Portulaca pilosa, Psilostrophe tagetina, Sporobolus cryptandrus, Thelesperma magapotamicum, Tridens muticus var. elongatus, and Yucca glauca. Two of these species, Phacelia integrifolia (Figure 4) and Nama stevensii (Figure 5 ), as well as the less-commonly encountered Haploesthes greggii (Figure 6), are found only on gypsum substrates in Oklahoma and are considered obligate gypsophiles. Two of the species in this habitat, Echinocereus reichenbachii (Figure 7) and Haploesthes greggii, are being tracked by the Oklahoma Natural Heritage Inventory. Woody species occurred mainly on the steep north-facing slopes and ravines of Cedar Canyon, and included Celtis laevigata var. reticulata, Cornus drummondii, Gleditsia triacanthos, Juniperus virginiana, Morus rubra, Rhus glabra, Rhus aromatica, Ribes aureum, Sapindus saponaria, Sideroxylon lanuginosum, Symphoricarpos orbiculatus, Ulmus americana, Ulmus rubra, and Vitis acerifolia.

\section{Wetland and riparian vegetation}

This vegetation was found along the banks of Cedar Creek as well as the margins of the pond. Associated species included Amorpha fruticosa, Baccharis salicina, Carex gravida, Eleocharis montevidensis, Equisetum spp., Nasturtium officinale, Pluchea odorata, Populus deltoides, Ranunculus sceleratus, Salix nigra, and Vitis riparia. A wet depression in the grassland on the north side of the canyon supported Marsilea vestita, a species tracked by the Oklahoma Natural Heritage Inventory.

\section{Disturbed areas and old-field vegetation}

This type of vegetation was found in disturbed areas along roadsides and trails near the visitor center, in campgrounds, and in areas with deeper soils north of the canyon that were grazed until 1997. Common species in disturbed areas along roadsides, trails, and campgrounds were Arenaria serpyllifolia, Bothriochloa ischaemum, Bromus spp., Chamaesaracha coniodes, Digitaria ciliaris, Erodium cicutarium, Glandularia pumila, Holosteum umbellatum, Lamium amplexicaule, Melilotus officinalis, Quincula lobata, Veronica spp., and Tribulus terrestris. Common species in old fields included Ambrosia psilostachya, Amphiachyris dracunculoides, Aristida oligantha, Artemisia ludoviciana, Bothriochloa laguroides, Bromus spp., Chamaesyce spp., and Gutierrezia sarothrae. Many of these species increase with grazing. Thickets of Prunus angustifolia were also present. 
season was provided by a mini-grant from the University of Central Oklahoma Joe Jackson College of Graduate Studies and Research. We also thank William Caire for his assistance with collections, and anonymous reviewers for constructive suggestions.

Table 1 Summary of floristic collections from Alabaster Caverns State Park in the Cimarron Gypsum Hills, Woodward County, Oklahoma*

\begin{tabular}{lccccc}
\hline Taxonomic Group & Families & Genera & Species & $\begin{array}{c}\text { Native } \\
\text { spp. }\end{array}$ & $\begin{array}{c}\text { Exotic } \\
\text { spp. }\end{array}$ \\
\hline $\begin{array}{l}\text { Monilophyta } \\
\text { Pinophyta }\end{array}$ & 3 & 4 & 4 & 4 & 0 \\
Magnoliophyta & 1 & 1 & 1 & 1 & 0 \\
Eudicots & 57 & 151 & 210 & 185 & 25 \\
Monocots & 5 & 43 & 59 & 42 & 17 \\
\hline Total & 66 & 199 & 274 & 232 & 42 \\
\hline
\end{tabular}

*Table format follows Palmer et al. (1995) 


\section{LITERATURE CITED}

Allen, L. 2007. Alabaster Caverns State Park. In Oklahoma Historical Society. Encyclopedia of Oklahoma History and Culture. [cited 2012 Jul 27]. Available from: http://digital.library.okstate.edu/encyclop edia/entries/A/AL002.html

Barkworth, M. E., L. K. Anderton, K. M. Capels, S. Long, and M. B. Piep, eds. 2007. Manual of Grasses for North America. Logan (UT): Intermountain Herbarium and Utah State University Press.

Buckallew, R. R. and G. M. Caddell. 2003. Vascular flora of the University of Central Oklahoma Selman Living Laboratory, Woodward County, Oklahoma. Proceedings of the Oklahoma Academy of Science. 83:31-45.

Buckallew, R. R. and G. M. Caddell. 2004. Erratum: Appendix 1: Taxa present at the SLL. Proceedings of the Oklahoma Academy of Science. 84:95-103.

Caywood, M. 2006. Park Manager, Alabaster Caverns State Park, Freedom, Oklahoma. Personal Communication.

Curtis, N. M., W. E. Ham, and K. S. Johnson. 2008. Geomorphic provinces of Oklahoma. In Johnson K.S. and K. V. Luza, eds. Earth Sciences and Mineral Resources of Oklahoma. Educational Publication 9, Oklahoma Geological Survey, The University of Oklahoma. Available from: http://www.ogs.ou.edu/pubsscanned/EP 9 All.pdf

Diggs, G. M., B. L. Lipscomb, and R. J. O'Kennon. Shinners and Mabler's Illustrated Flora of North Central Texas. Fort Worth: Botanical Research Institute of Texas.

Duck, L. G. and J. D. Fletcher. 1943. A game type map of Oklahoma. In A survey of the game and furbearing animals of Oklahoma. Oklahoma Department of Wildlife Conservation, Oklahoma City. Available from: http://biosurvey.ou.edu/download/duckf lt/dfmap.gif
Great Plains Flora Association. 1986. Flora of the Great Plains. Lawrence: University of Kansas.

Hitchcock, A. S. 1971. Manual of the Grasses of the United States. Toronto, Ontario: General Publishing Co.

Hoagland, B. W. 2000. The vegetation of Oklahoma: A classification for landscape mapping and conservation planning. Southwestern Naturalist 45:385-420.

Hoagland, B. W. and A. K. Buthod. 2005. Vascular flora of a gypsum dominated site in Major County, Oklahoma. Proceedings of the Oklahoma Academy of Science. 85:1-8.

Nance, E. C., C. A. Steers, E. L. Cole, M. L. Miller, and C. Fanning. 1963. Soil survey of Woodward County. United States Department of Agriculture, Soil Conservation Service.

Meyers, A. J., A. M. Gibson, B. P. Glass, and C. R. Patrick. 1969. Guide to Alabaster Cavern, Woodward County, Oklahoma. Oklahoma Geological Survey Guidebook No. 15, The University of Oklahoma, Norman.

Oklahoma Climatological Survey http://climate.ok.gov/ [cited 2012 Sep 2012]. Available from: http://climate.ok.gov/county climate/Pr oducts/County Climatologies/county cli mate woodward.pdf

Oklahoma Invasive Plant Council. 2012. Oklahoma Non-Native Invasive Plant Species. [cited 2012 Sep 9]. Available from: http:/ok-invasive-plantcouncil.org/images/OKinvasivespp.pdf

Oklahoma Natural Heritage Inventory (ONHI). 2012. Oklahoma Natural Heritage Inventory Plant Tracking List. Available from: http://www.biosurvey.ou.edu/download/ publications/onhi plants tracking 52012. pdf

Palmer, M. W., G. L. Wade, and P. R. Neal. 1995. Standards for the writing of floras. Bioscience 45:339-345.

Stevens, P. F. 2012. Angiosperm Phylogeny Website. Version 12 [cited 2012 Jul].

(C) Gloria M. Caddell, Kristi D. Rice Journal compilation (C) 2012 Oklahoma Native Plant Society 
Murray, A. K. Ryburn, B. A. Smith, C. E. S. Taylor, R. A. Thompson, J. B. Walker, and L. E. Watson. 2005, 2010. Keys and Descriptions for the V ascular Plants of Oklahoma. Noble (OK): Flora Oklahoma Inc.

USDA, NRCS. 2012. The PLANTS Database National Plant Data Team, Greensboro, NC 27401-4901 USA. [cited 2012 Sep] http://plants.usda.gov. 


\begin{abstract}
APPENDIX
Annotated species list for Alabaster Caverns State Park, Woodward County, Oklahoma. Nomenclature and common names are based on USDA, NRCS (2012). Organization of taxa is based on Angiosperm Phylogeny Group (APG III) recommendations (Stevens 2012). Life history (A=annual, B=biennial, $P=$ perennial) and collection numbers follow the species names. Taxa introduced to North America are indicated with an asterisk $\left(^{*}\right)$ and those on the Oklahoma Natural Heritage Inventory Plant Tracking List are indicated with a symbol $(+)$. Voucher specimens were deposited in the University of Central Oklahoma Herbarium (CSU).
\end{abstract}

\title{
MONILOPHYTA
}

Equisetaceae

Equisetum L sp. (horsetail) - P; GMC1215

\section{Marsileaceae}

+Marsilea vestita Hook \& Grev. (hairy waterclover) - P; GMC1145

\section{Pteridaceae}

Cheilanthes feei T. Moore (slender lipfern) - P; GMC800

Pellaea atropurpurea (L.) Link (purple cliffbreak) - P; GMC815

\section{GYMNOSPERMS/PINOPHYTA}

\section{Cupressaceae}

Juniperus virginiana L. var. virginiana (eastern redcedar) - P; GMC816

\section{ANGIOSPERMS/MAGNOLIOPHYTA EUDICOTS \\ Amaranthaceae \\ Amaranthus tuberculatus (Moq.) Sauer (roughfruit amaranth) - A; GMC1245 \\ ${ }^{*}$ Chenopodium album L. var. album (lambsquarters) - A; KR930 \\ Chenopodium berlandieri Moq. (pitseed goosefoot) - A; GMC1217}

\section{Anacardiaceae}

Rhus aromatica Aiton - P; GMC811

Rhus copallinum L. (winged sumac) - P; GMC1177

Rhus glabra L. (smooth sumac) - P; GMC849

Toxicodendron radicans (L.) Kuntze (eastern poison ivy) - P; GMC1267

\section{Apiaceae}

Ammoselinum popei Torr. \& A. Gray (plains sandparsley) - A; KR753

Sanicula canadensis L. (Canadian blacksnakeroot) - B; GMC1170

Spermolepis inermis (Nutt. ex DC.) Mathias \& Constance (Red River scaleseed) - A; GMC1165

\section{Apocynaceae}

Apocynum cannabinum L. (Indianhemp) - P; GMC 1137

Asclepias asperula (Decne.) Woodson ssp. capricornu (Woodson) Woodson

(antelopehorns) - P; GMC1096 
Asclepias engelmanniana Woodson (Engelmann's milkweed) - P; GMC1186

Asclepias latifolia (Torr.) Raf. (broadleaf milkweed) - P; GMC1189

Asclepias viridiflora Raf. (green comet milkweed) - P; GMC870

Asclepias viridis Walter (green antelopehorn) - P; GMC1136

\section{Asteraceae}

Achillea millefolium L. (common yarrow) - P; GMC1107

Ambrosia psilostachya DC. (Cuman ragweed) - P; GMC897

Ambrosia trifida L. (great ragweed) - A; GMC914

Amphiachyris dracunculoides (DC.) Nutt. (prairie broomweed) - A; GMC922

Artemisia dracunculus L. (tarragon) - P; GMC921

Artemisia filifolia Torr. (sand sagebrush) - P; GMC895

Artemisia ludoviciana Nutt. ssp. Iudoviciana (white sagebrush) - P; GMC941

Baccharis salicina Torrey \& A. Gray (willow baccharis) - P; GMC901

Brickellia eupatorioides (L.) Shinners var. corymbulosa (Torr. \& A. Gray) Shinners

(false boneset) - P; GMC1242

Chaetopappa ericoides (Torr.) G. L. Nesom (rose heath) - P; GMC1063

Cirsium undulatum (Nutt.) Spreng. (wavy leaf thistle) - P; GMC1161

Conyza canadensis (L.) Cronquist (Canadian horseweed) - A; KR929

Conyza ramosissima Cronquist (dwarf horseweed) - A; GMC1256

Echinacea angustifolia DC. (blacksamson echinacea) - P; GMC1136

Erigeron cf. divergens Torr. \& A. Gray (spreading fleabane) - B; GMC973

Erigeron strigosus Muhl. ex Willd. (prairie fleabane) - A; GMC1097

Evax prolifera Nutt. ex DC. (bighead pygmycudweed) - A; GMC1030

Gaillardia pulchella Foug. (Indian blanket) - A; GMC828

Gaillardia suavis (A. Gray \& Engelm.) Britton \& Rusby (perfumeballs) - P; GMC1133

Grindelia papposa G. L. Nesom and Suh (Spanish gold) - A; GMC1203

Grindelia squarrosa (Pursh) Dunal (curlycup gumweed) - B; GMC935

Gutierrezia sarothrae (Pursh) Britton \& Rusby (broom snakeroot) - P; GMC907

+ Haploesthes greggii A. Gray (false broomweed) - P; GMC1147

Helianthus annuus L. (common sunflower) - A; GMC854

Helianthus petiolaris Nutt. (prairie sunflower) - A; GMC1244

Heterotheca stenophylla (A. Gray) Shinners (stiffleaf false goldenaster) - P; GMC891

Hymenopappus tenuifolius Pursh (Chalk Hill hymenopappus) - B; GMC1128

Iva annua L. (annual marshelder) - A; GMC1257

Lactuca Iudoviciana (Nutt.) Riddell (biannual lettuce) - B; GMC814

Liatris punctata Hook. (dotted blazing star) - P; GMC926

Machaeranthera pinnatifida (Hook.) Shinners (tansyaster) - P; GMC1160

Packera plattensis (Nutt.) W.A. Weber \& Á. Löve (prairie groundsel) - B, P; GMC1104

Pluchea odorata (L.) Cass. (sweetscent) - A; GMC1251

Psilostrophe tagetina (Nutt.) Greene var. cerifera (A. Nelson) B. L. Turner (woolly paperflower) - P; GMC843

Pyrrhopappus grandiflorus (Nutt.) Nutt. (tuberous desert-chicory) - P; GMC1005

Ratibida columnifera (Nutt.) Woot. \& Standl. (upright prairie coneflower) - P; GMC1113

Senecio riddellii Torr. \& A. Gray (Riddell's ragweed) - P

Solidago missouriensis Nutt. var. fasciculata Holz (Missouri goldenrod) - P; GMC1220

Solidago petiolaris Aiton (downy ragged goldenrod) - P; GMC908

(C) Gloria M. Caddell, Kristi D. Rice

Journal compilation (C) 2012 Oklahoma Native Plant Society 
*Sonchus asper (L.) Hill (spiny sowthistle) - A; GMC1142

Symphyotrichum ericoides (L.) G. L. Nesom (white heath aster) - P; GMC944

*Taraxacum officinale F. H. Wigg (common dandelion) - P; GMC822

Tetraneuris scaposa (DC.) Greene (stemmy four-nerve daisy) - P; GMC1053

Thelesperma megapotamicum (Spreng.) Kuntze (Hopi tea greenthread) - P; GMC803

*Tragopogon dubius Scop. (yellow salsify) - B; GMC1143

Vernonia baldwinii Torr. (Baldwin's ironweed) - P; GMC900, GMC864

Xanthium strumarium L. var. canadense (Mill.) Torr. \& Gray (Canada cocklebur) - A; GMC1253

\section{Boraginaceae}

Lappula occidentalis (S. Watson) Greene (flatspine stickseed) - A; KR752

Lithospermum incisum Lehm. (narrowleaf stoneseed) - P; GMC968

\section{Brassicaceae}

${ }^{*}$ Camelina rumelica Velen. (graceful false flax) - A; GMC1089

${ }^{*}$ Capsella bursa-pastoris (L.) Medik. (shepherd's purse) - A; GMC979

Descurainia pinnata (Walter) Britton (western tansymustard) - A; GMC1031

Draba reptans (Lam.) Fernald (Carolina draba) - A; GMC965

Lepidium densiflorum Schrad. (common pepperweed) - A; GMC1086

Lepidium oblongum Small (veiny pepperweed) - A, B; GMC1081, GMC963

Lesquerella gordonii (A. Gray) S. Watson (Gordon's bladderpod) - A; GMC966, GMC1057

*Nasturtium officinale W.T. Aiton (watercress) - P; GMC1179

\section{Cactaceae}

Cylindropuntia imbricata (Haw.) F.M.Knuth (tree cholla) - P

Echinocereus reichenbachii (Terscheck ex Walp.) hort ex Haage (lace hedgehog cactus) - $\mathrm{P}$

Escobaria missouriensis (Sweet) D.R. Hunt (Missouri foxtail cactus) - P; GMC1195

Escobaria vivipara (Nutt.) Buxbaum var. vivipara (spinystar) - P; GMC1164

Opuntia phaeacantha Engelm. (tulip pricklypear) - P; GMC1144

\section{Campanulaceae}

Triodanis perfoliata (L.) Nieuwl. (clasping Venus' looking-glass) - A; GMC1122

\section{Cannabaceae}

Celtis laevigata Willd. var. laevigata (sugarberry) - P; GMC1071

Celtis laevigata Willd. var. reticulata (Torr.) L.D. Benson (netleaf hackberry) - P; GMC917

Celtis occidentalis L. (common hackberry) - P; GMC804

\section{Caprifoliaceae}

Symphoricarpos orbiculatus Moench (coralberry) - P; GMC915

\section{Caryophyllaceae}

*Arenaria serpyllifolia L. (thymeleaf sandwort) - A; GMC821

Cerastium nutans Raf. (nodding chickweed) - A; GMC1064

${ }^{*}$ Cerastium pumilum W. Curtis (European chickweed) - A; GMC1039

*Holosteum umbellatum L. (jagged chickweed) - A; GMC976

Paronychia jamesii Torr. \& A. Gray (James' nailwort) - P; GMC883 
Silene antirrhina L. (sleepy silene) - A; GMC1123

*Stellaria media (L.) Vill. ssp. pallida (Dumort) Asch. \& Graebon. (common chickweed) - A; GMC1014

\section{Celastraceae}

Celastrus scandens L. (American bittersweet) - P; GMC1002

\section{Cleomaceae}

Polanisia dodecandra (L.) DC. (redwhisker clammyweed) - A; GMC868

\section{Clusiaceae}

*Hypericum perforatum L. (common St. Johnswort) - P; GMC1166

\section{Convolvulaceae}

Evolvulus nuttallianus Schult. (shaggy dwarf morning-glory) - P; GMC1088

Ipomoea leptophylla Torr (bush morning-glory) - P; GMC1146

\section{Cornaceae}

Cornus drummondii C.A. Mey. (roughleaf dogwood) - P; GMC799

\section{Cucurbitaceae}

Cucurbita foetidissima Kunth (Missouri gourd) - P; GMC1172

\section{Euphorbiaceae}

Acalypha ostryifolia Riddell (pineland threeseed mercury) - A; GMC927

Chamaesyce stictospora (Engelm.) Small (slimseed sandmat) - A; GMC892

Chamaesyce glyptosperma (Engelm.) Small (ribseed sandmat) - A; GMC1219

Chamaesyce maculata (L.) Small (spotted sandmat) - A; GMC1241

Chamaesyce missurica (Raf.) Shinners (prairie sandmat) - A; GMC869

Chamaesyce serpens (Kunth) Small (matted sandmat) - A; GMC1259

Croton monanthogynus Michx. (prairie tea) - A; GMC930

Croton texensis (Klotzsch) Mull. Arg. (Texas croton) - A; GMC886, GMC862, GMC902

Euphorbia dentata Michx. (toothed spurge) - A; GMC953

Euphorbia marginata Pursh (snow on the mountain) - A; GMC937

Euphorbia spathulata Lam. (warty spurge) - A; GMC1060

\section{Fabaceae}

Amorpha canescens Pursh (leadplant) - P; GMC825

Amorpha fruticosa $L$. (false indigo bush) - P; GMC840

Astragalus gracilis Nutt. (slender milkvetch) - P; GMC993

Astragalus lotiflorus Hook. (lotus milkvetch) - P; GMC967, GMC992

Astragalus missouriensis Nutt. (Missouri milkvetch) - P; GMC1092, GMC1269, GMC969

Astragalus mollissimus Torr. (woolly locoweed) - P; GMC1093

Astragalus nuttallianus DC. var. austrinus (Small) Barneby (smallflowered milkvetch) - A; GMC1049

Astragalus plattensis Nutt. (Platte River milkvetch) - P; GMC1046, GMC1047, GMC1099

Dalea aurea Nutt. ex Pursh (golden prairie clover) - P; GMC863

Dalea candida Michx. ex Willd. var. candida (white prairie clover) - P; GMC866

Dalea enneandra Nutt. (nineanther prairie clover) - P; GMC1154 
Dalea purpurea Vent. (purple prairie clover) - P; GMC1153

Desmanthus illinoensis (Michx.) MacMill. ex B.L Rob. \& Fernald (Illinois bundleflower) - P; GMC924

Gleditsia triacanthos L (honeylocust) - P; GMC986

*Medicago minima (L.) L. (little bur-clover) - A; GMC1043

*Melilotus officinalis (L.) Lam. (sweetclover) - A,B; GMC827, GMC850

Mimosa quadrivalvis L. (fourvalve mimosa) - P; GMC1090

Pediomelum cuspidatum (Pursh) Rydb. (largebract Indian breadroot) - P; GMC1091, GMC 1135

Prosopis glandulosa Torr. (honey mesquite) - P; GMC932

Psoralidium tenuiflorum (Pursh) Rydb. (slimflower scurfpea) - P; GMC1169

Robinia pseudoacacia L. (black locust) - P; GMC1070

Vicia americana Muhl. ex Willd. (American vetch) - P; GMC1000

Vicia ludoviciana Nutt. (Louisiana vetch) - A; GMC1094

\section{Fagaceae}

Quercus muehlenbergii Engelm. (chinkapin oak) - P

\section{Geraniaceae}

*Erodium cicutarium (L.) L'Her. ex Aiton (redstem stork's bill) - A; GMC 836

*Geranium pusillum L. (small geranium) - A; 1020

\section{Grossulariaceae}

Ribes aureum Pursh var. villosum DC. (golden currant) - P; GMC971

\section{Hydrophyllaceae}

Nama stevensii C.L. Hitchc. (Steven's fiddleleaf) - A; GMC1041

Phacelia integrifolia Torr. (gyp phacelia) - A,B; GMC1187

\section{Lamiaceae}

Hedeoma hispida Pursh (rough false pennyroyal) - A; GMC795

*Lamium amplexicaule L. (henbit deadnettle) - A; GMC981

Monarda clinopodioides A. Gray (basil beebalm) - A; GMC1159

Teucrium laciniatum Torr. (lacy germander) - P; GMC1134

\section{Linaceae}

Linum pratense (Norton) Small (meadow flax) - A; GMC1066

Linum rigidum Pursh (stiffstem flax) - A; GMC1067

\section{Loasaceae}

Mentzelia nuda (Pursh) Torr. \& A. Gray var. stricta (Osterh.) Harrington (bractless blazingstar) - B,P; GMC1188

Mentzelia oligosperma Nutt. ex Sims (chickenthief) - P; GMC802

\section{Malvaceae}

Callirhoe involucrata (Torr. \& A. Gray) A. Gray (purple poppymallow) - P; GMC1006 Sphaeralcea coccinea (Nutt.) Rydb. (scarlet globemallow) - P; GMC1051 


\section{Molluginaceae}

Mollugo verticillata L. (green carpetweed) - A; GMC1229

\section{Moraceae}

Morus rubra L (red mulberry) - P: GMC1138

\section{Nyctaginaceae}

Mirabilis linearis (Pursh) Heimerl (narrowleaf four o'clock) - P; GMC1180

Mirabilis nyctaginea (Michx.) MacMill. (heartleaf four o'clock) - P; GMC1139

\section{Oleaceae}

Forestiera pubescens Nutt. (stretchberry) - P; GMC1249

\section{Onagraceae}

Oenothera cinerea (Wooton \& Standl.) W.L. Wagner \& Hoch (woolly beeblossom) - P; GMC809

Oenothera curtiflora W.L. Wagner \& Hoch (velvetweed) - A; GMC1148

Oenothera glaucifolia W.L. Wagner \& Hoch (false gaura) - P; GMC807

Oenothera hartwegii Benth. (Hartweg's sundrops) - P; GMC1127

Oenothera serrulata Nuttall (yellow sundrops) - P; GMC1110

Oenothera suffrutescens (Ser.) W.L. Wagner \& Hoch (scarlet beeblossom) - P; GMC1052, GMC1126

\section{Orobanchaceae}

Agalinis aspera (Douglas ex Benth.) Britton (tall false foxglove) - A; GMC1228

Castilleja purpurea (Nutt.) G. Don var. citrina (Pennell) Shinners (prairie Indian paintbrush) - P; GMC991

Orobanche ludoviciana Nutt. ssp. multiflora (Nutt.) T.S. Collins ex H.L. White \& W.C.

Holmes (manyflower broomrape) - A; GMC1196

\section{Oxalidaceae}

Oxalis corniculata L. (creeping woodsorrel) - A; GMC983

Oxalis dillenii Jacq. (slender yellow woodsorrel) - P; GMC1019

\section{Papaveraceae}

Argemone polyanthemos (Fedde) G.B. Ownbey (crested pricklypoppy) - A

Corydalis micrantha (Engelm. ex A. Gray) A. Gray (smallflower fumewort) - A; GMC1271

\section{Plantaginaceae}

Nuttallanthus canadensis (L.) D.A. Sutton (Canada toadflax) - A; KR441

Penstemon cobaea Nutt. (cobaea beardtongue) - P; GMC1056

Plantago patagonica Jacq. (woolly plantain) - A; GMC1087

Plantago rhodosperma Decne. (redseed plantain) - A; GMC1062

*Veronica arvensis L. (corn speedwell) - A; GMC1045

Veronica peregrina L. ssp. xalapensis (Kunth) Pennell (hairy purslane speedwell) - A; GMC964

*Veronica polita Fr. (gray field speedwell) - A; GMC 984

\section{Polygalaceae}

Polygala alba Nutt. (white milkwort) - P; GMC865 


\section{Polygonaceae}

*Polygonum persicaria L. (spotted ladysthumb) - A; GMC1190

Polygonum ramosissimum Michx. (bushy knotweed) - A; GMC1191

Rumex altissimus Alph. Wood (pale dock) - P; GMC1209

\section{Portulacaceae}

${ }^{*}$ Portulaca oleracea L. (little hogweed) - A; GMC1232

Portulaca pilosa L. (kiss me quick) - A; GMC925

\section{Primulaceae}

Androsace occidentalis Pursh (western rockjasmine) - A; GMC1272

\section{Ranunculaceae}

Delphinium carolinianum Walter ssp. virescens (Nutt.) R.E. Brooks (Carolina larkspur) - P

Ranunculus sceleratus L. (cursed buttercup) - A

\section{Rhamnaceae}

Ceanothus herbaceus Raf. (Jersey tea) - P; GMC1106

\section{Rosaceae}

Prunus angustifolia Marsh. (Chickasaw plum) - P; GMC844, GMC972

\section{Rubiaceae}

Galium aparine L. (stickywilly) - A; GMC1003

Galium circaezans Michx. (licorice bedstraw) - P; GMC1193

Stenaria nigricans (Lam.) Terrell var. nigricans (prairie bluet) - P; GMC1167

\section{Salicaceae}

Populus deltoides Bartram ex Marsh. (eastern cottonwood) - P

Salix nigra Marsh. (black willow) - P; GMC997

\section{Sapindaceae}

Sapindus saponaria L. var. drummondii (Hook. and Arn.) L.D. Benson (western soapberry) - P; GMC1206

\section{Sapotaceae}

Sideroxylon lanuginosum Michx. (gum bully) - P; GMC835, GMC1207

\section{Simaroubaceae}

${ }^{*}$ Ailanthus altissima (Mill.) Swingle (tree of heaven) - P

\section{Solanaceae}

Chamaesaracha coniodes (Moric. ex Dunal) Britton (gray five eyes) - P; GMC1044

Physalis cf. hederifolia A. Gray (ivyleaf groundcherry) - P; GMC857

Physalis longifolia Nutt. (longleaf groundcherry) - P; GMC1205

Physalis mollis Nutt. (field groundcherry) - P; GMC1216

Quincula lobata (Torr.) Raf. (Chinese lantern) - P; GMC1085 
Solanum elaeagnifolium Cav. (silverleaf nightshade) - P; GMC896

Solanum rostratum Dunal (buffalobur nightshade) - A; GMC936

\section{Tamaricaceae}

*Tamarix ramosissima Ledeb. (saltcedar) - P; GMC1192

\section{Ulmaceae}

Ulmus americana L. (American elm) - P; GMC970

*Ulmus pumila L. (Siberian elm) - P; GMC978

Ulmus rubra Muhl. (slippery elm) - $P$

\section{Urticaceae}

Parietaria pensylvanica Muhl. ex Willd. (Pennsylvania pellitory) - A

\section{Verbenaceae}

Glandularia bipinnatifida (Nutt.) Nutt. (Dakota mock vervain) - P; GMC1050

Glandularia pumila (Rydb.) Umber (pink mock vervain) - A; GMC830

\section{Violaceae}

Viola bicolor Pursh (field pansy) - A; GMC962

\section{Vitaceae}

Cissus trifoliata (L.) L. (sorrelvine) - P; GMC845

Parthenocissus quinquefolia (L.) Planch. (Virginia creeper) - P; GMC823

Vitis acerifolia Raf. (mapleleaf grape) - P; GMC1175

Vitis riparia Michx. (riverbank grape) - P; GMC826, GMC1208

\section{Zygophyllaceae}

*Tribulus terrestris L. (puncturevine) - A; GMC1198

\section{MONOCOTS}

Amaryllidaceae

Allium drummondii Regel (Drummond's onion) - P; GMC987

\section{Asparagaceae}

Androstephium coeruleum (Scheele) Greene (blue funnel lily) - P; GMC974 Yucca glauca Nutt. var. glauca (soapweed yucca) - P; GMC1061

\section{Commelinaceae}

Tradescantia occidentalis (Britton) Smyth (prairie spiderwort) - P; GMC1095

\section{Cyperaceae}

Carex gravida L.H. Bailey (heavy sedge) - P; GMC838

Cyperus lupulinus (Spreng.) Marcks (Great Plains flatsedge) - P; GMC929

Eleocharis montevidensis Kunth (sand spikerush) - P; GMC1273 


\section{Poaceae}

${ }^{*}$ Aegilops cylindrica Host (jointed goatgrass) - A; GMC1108

Andropogon hallii Hack. (sand bluestem) - P; GMC950

Aristida oligantha Michx. (prairie threeawn) - A; GMC1221, GMC1262

Aristida purpurea Nutt. (purple threeawn) - P; GMC861

*Bothriochloa ischaemum (L.) Keng (yellow bluestem) - P; GMC955

Bothriochloa laguroides (DC.) Herter ssp. torreyana (Steud.) Allred \& Gould (silver beardgrass) - P; GMC1162

Bouteloua curtipendula (Michx.) Torr. (sideoats grama) - P; GMC846

Bouteloua gracilis (Willd. ex Kunth) Lag. ex Griffiths (blue grama) - P; GMC872

Bouteloua hirsuta Lag. (hairy grama) - P; GMC884

*Bromus catharticus Vahl (rescuegrass) - A; GMC989

${ }^{*}$ Bromus arvensis L. (field brome) - A; GMC1119

${ }^{*}$ Bromus tectorum L. (cheatgrass) - A; GMC1124, GMC988

Buchloe dactyloides (Nutt.) J.T. Columbus (buffalograss) - P; GMC1116, GMC1027, GMC960

Cenchrus spinifex Cav. (coastal sandbur) - A; GMC834

Chloris verticillata Nutt. (tumble windmill grass) - P; GMC1231

${ }^{*}$ Dactylis glomerata L. (orchardgrass) - P; GMC1140

Dichanthelium oligosanthes (Schult.) Gould var. scribnerianum (Nash) Gould (Scribner's

rosette grass) - P; GMC1101, GMC1152

Digitaria ciliaris (Retz.) Koeler (southern crabgrass) - A; GMC1230, GMC1255

Echinochloa muricata (P. Beauv.) Fernald (rough barnyardgrass) - A; GMC1264

*Eleusine indica (L.) Gaertn. (Indian goosegrass) - A; GMC1254

Elymus canadensis L. (Canada wildrye) - P; GMC1155

Elymus virginicus L. (Virginia wildrye) - P; GMC1210

${ }^{\star}$ Eragrostis cilianensis (All.) Vign. ex Janchen (stinkgrass) - A; GMC1222

Eragrostis secundiflora J. Presl ssp. oxylepis (Torr.) S.D. Koch (red lovegrass) - P; GMC920

Eragrostis spectabilis (Pursh) Steud. (purple lovegrass) - P; GMC943

Erioneuron pilosum (Buckley) Nash (hairy woollygrass) - P; KR404

Hordeum pusillum Nutt. (little barley) - A; GMC1102, GMC791

*Lolium perenne L. (perennial ryegrass) - P; GMC788

Muhlenbergia racemosa (Michx.) Britton, Sterns \& Poggenb. (marsh muhly) - P; GMC904

Panicum capillare L. (witchgrass) - A; GMC1218, GMC856

Panicum obtusum Kunth (vine mesquite) - P; GMC1248, GMC946

Panicum virgatum L. (switchgrass) - P; GMC874

Pascopyrum smithii (Rydb.) Á. Löve (western wheatgrass) - P; GMC1129

Phalaris caroliniana Walter (Carolina canarygrass) - A; GMC1083

*Poa annua L. (annual bluegrass) - A; GMC980

Poa arida Vasey (plains bluegrass) - P; GMC1018

*Poa pratensis L. (Kentucky bluegrass) - P; GMC990, GMC1007, GMC790, GMC1022

*Schedonorus phoenix (Scop.) Holub (tall fescue) - P; GMC1021

Sclerochloa dura (L.) P. Baeuv. (common hardgrass) - A; GMC977

Schizachyrium scoparium (Michx.) Nash (little bluestem) - P; GMC940

*Secale cereale L. (cereal rye) - A; GMC1011

*Setaria pumila (Poir.) Roem. \& Schult. (yellow foxtail) - A; GMC1240

*Setaria viridis (L.) P. Beauv. (green bristlegrass) - A; GMC911, GMC1199

Sorghastrum nutans (L.) Nash (Indiangrass) - P; GMC898

(C) Gloria M. Caddell, Kristi D. Rice

Journal compilation (C) 2012 Oklahoma Native Plant Society 
*Sorghum halepense (L.) Pers. (Johnsongrass) - P; GMC824, GMC912

Sporobolus compositus (Poir.) Merr. var. compositus (composite dropseed) - P; GMC931, GMC1223

Sporobolus cryptandrus (Torr.) A. Gray (sand dropseed) - P; GMC876, GMC1225, GMC1234

*Thinopyrum ponticum (Podp.) Z.-W. Liu \& R.-C. Wang (tall wheatgrass) - P; GMC1265

Tridens flavus (L.) Hitchc. (purpletop tridens) - P; GMC1213

Tridens muticus (Torr.) Nash var. elongatus (Buckley) Shinners (slim tridens) - P; GMC 1224, GMC1233

Tripsacum dactyloides (L.) L. (eastern gamagrass) - P; GMC847, GMC1184

Vulpia octoflora (Walter) Rydb. (sixweeks fescue) - A; GMC1033, GMC994

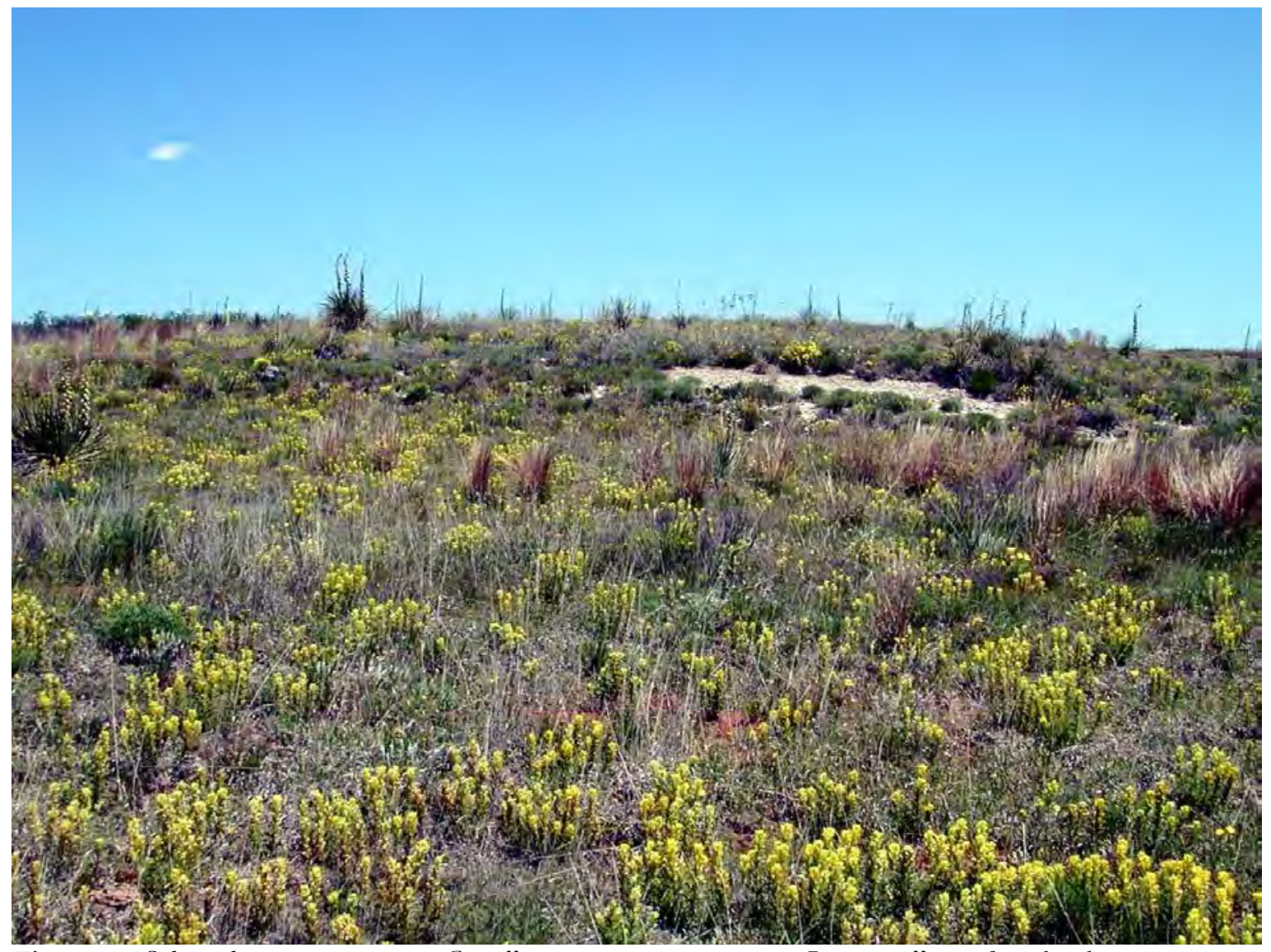

Figure 1 Schizachyrium scoparium-Castilleja purpurea var. citrina-Lesquerella gordonii herbaceous association on gypsum at Alabaster Caverns State Park. Photo courtesy of William Caire. 


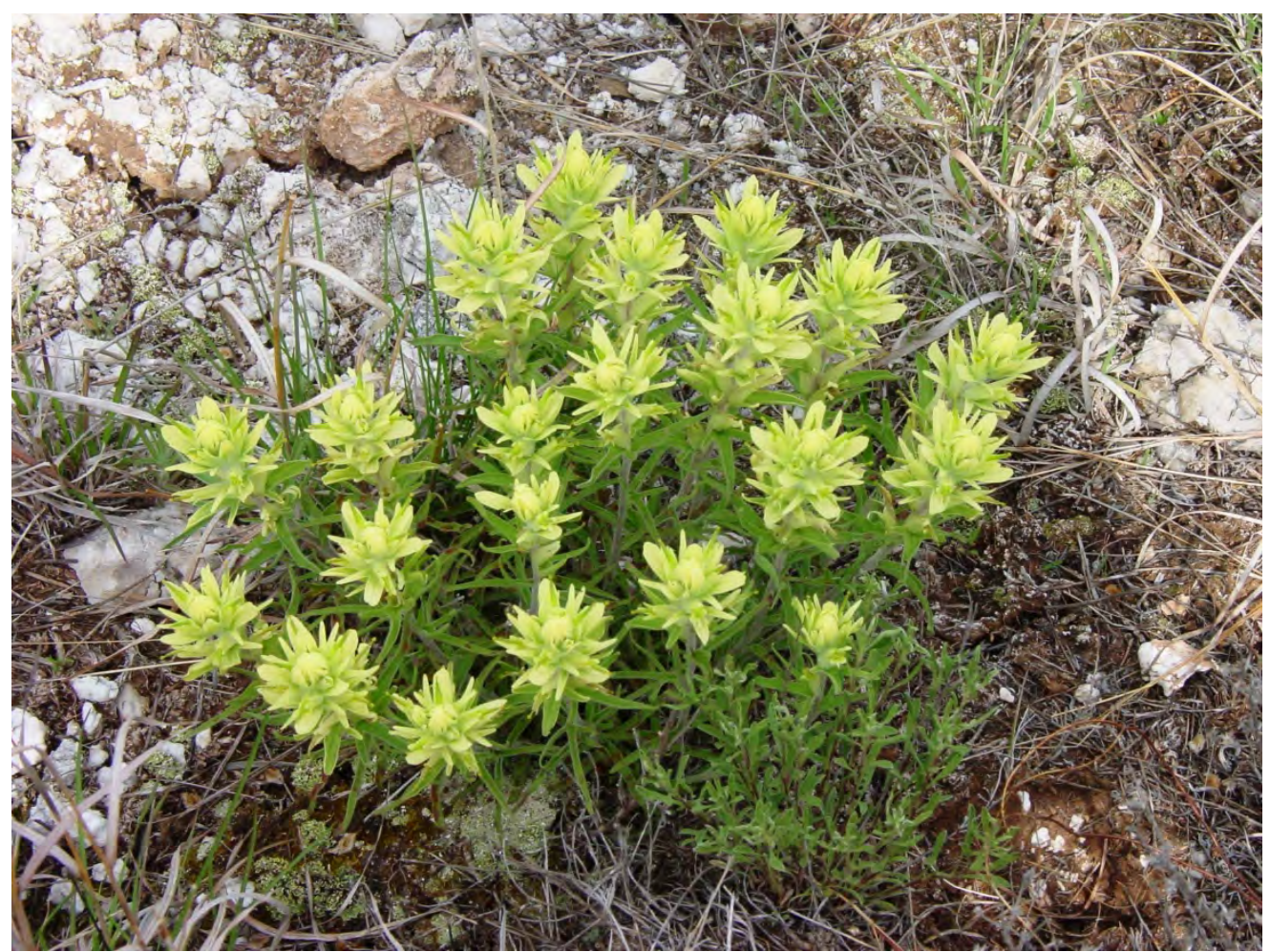

Figure 2 Castilleja purpurea var. citrina on gypsum outcrop at Alabaster Caverns State Park. Photo by G. Caddell.

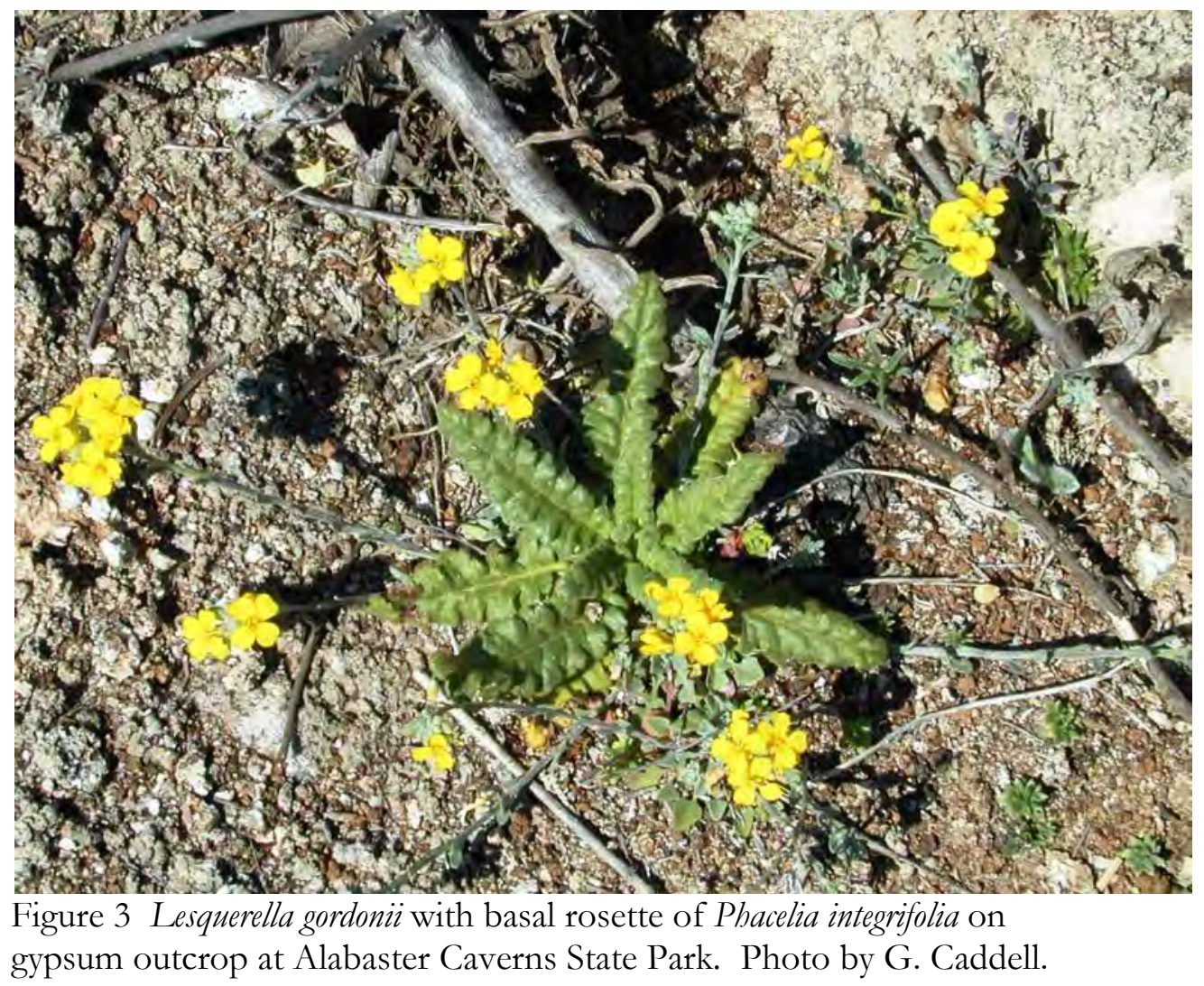

(C) Gloria M. Caddell, Kristi D. Rice

Journal compilation (C) 2012 Oklahoma Native Plant Society 


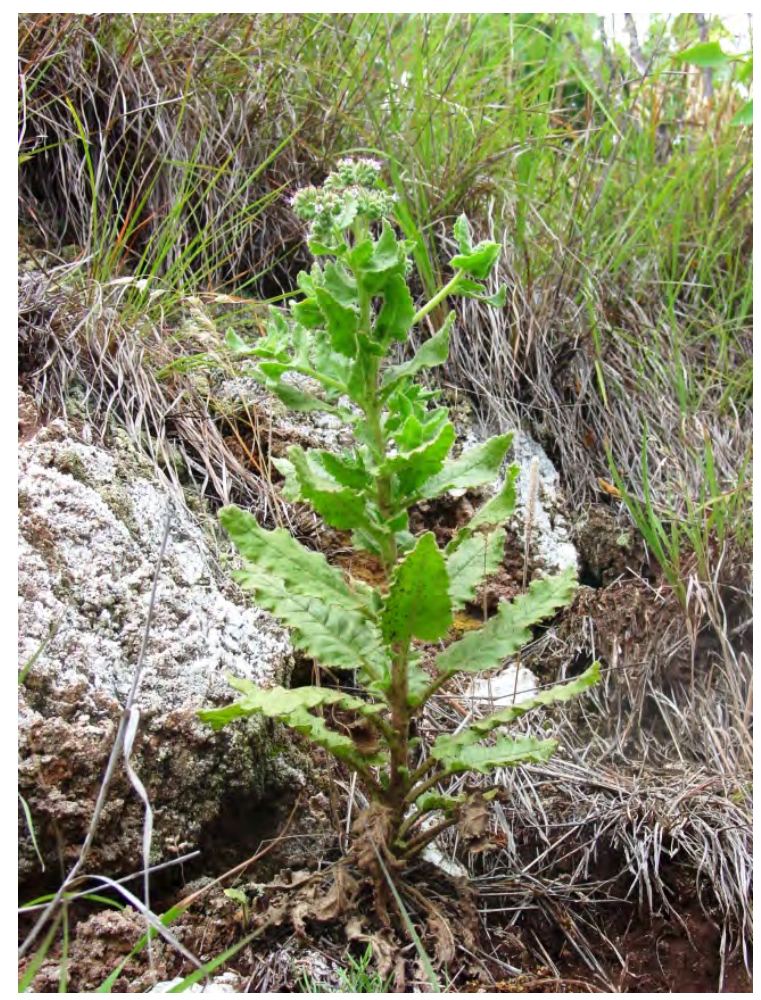

Figure 4 Phacelia integrifolia, an obligate

gypsophile, at Alabaster Caverns State Park.

Photo by G. Caddell.

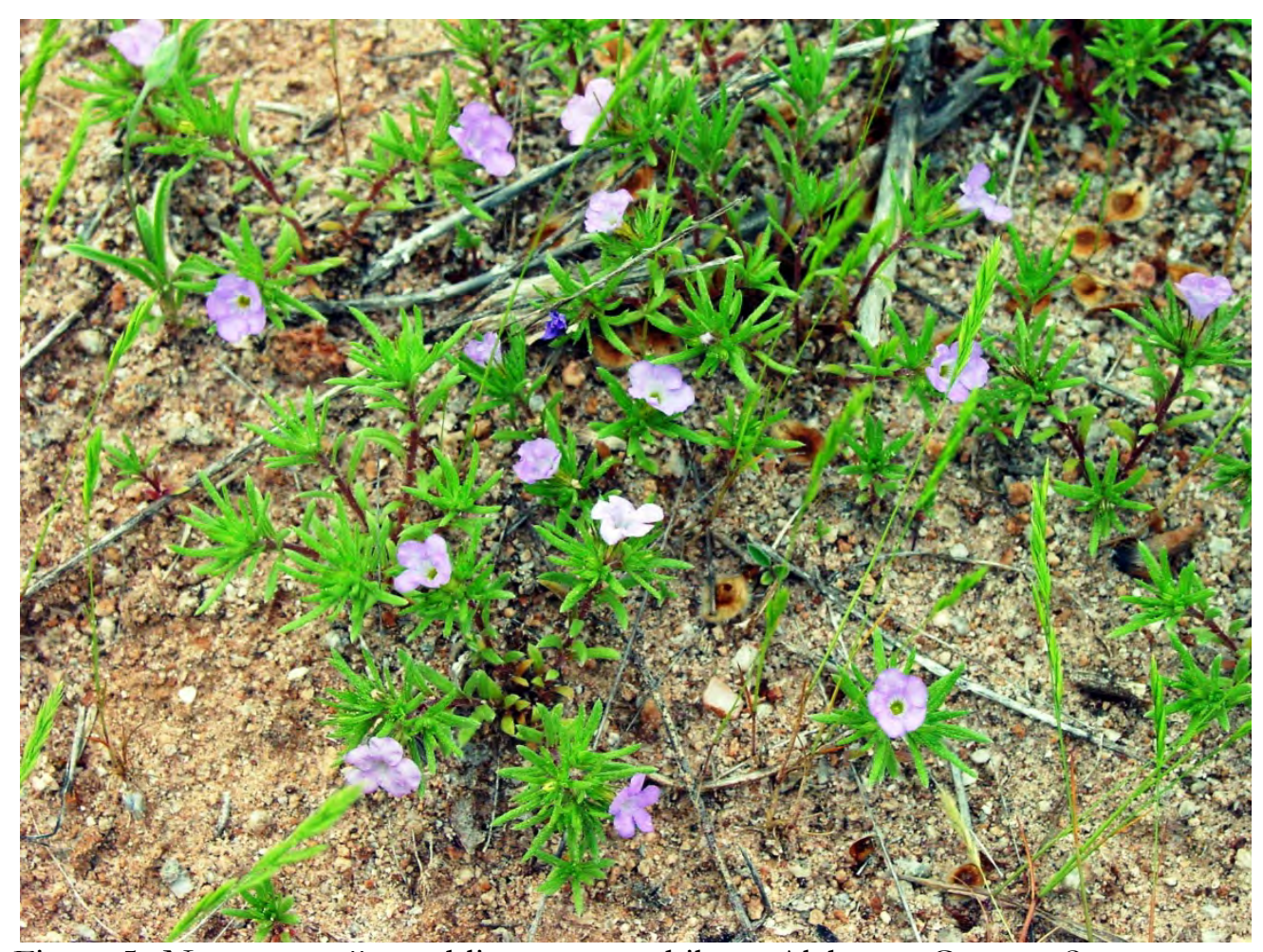

Figure 5 Nama stevensii, an obligate gypsophile, at Alabaster Caverns State

Park. Photo by G. Caddell.

(C) Gloria M. Caddell, Kristi D. Rice

Journal compilation C 2012 Oklahoma Native Plant Society 


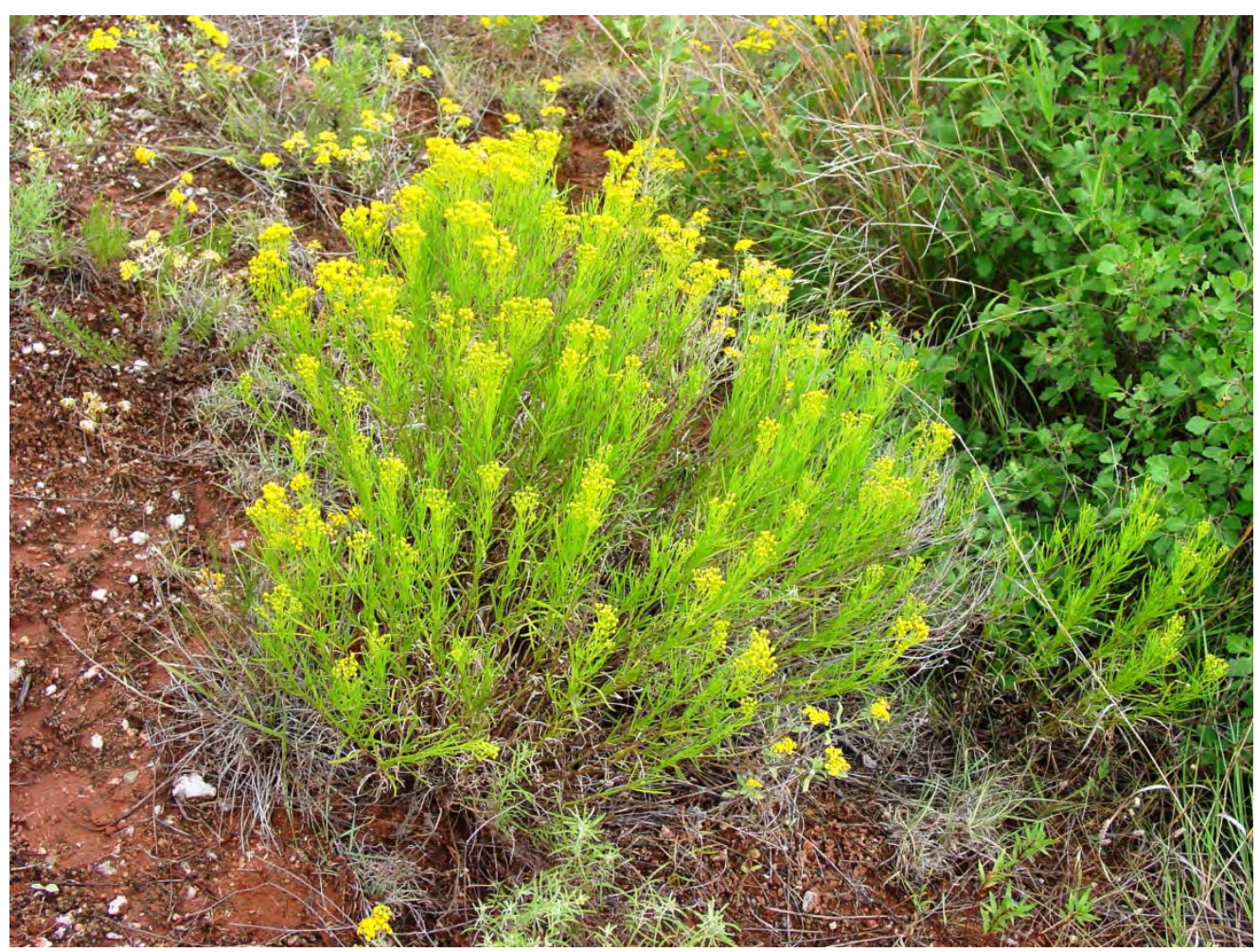

Figure 6 Haploesthes greggii, an obligate gypsophile, at Alabaster Caverns State Park. Photo by G. Caddell.

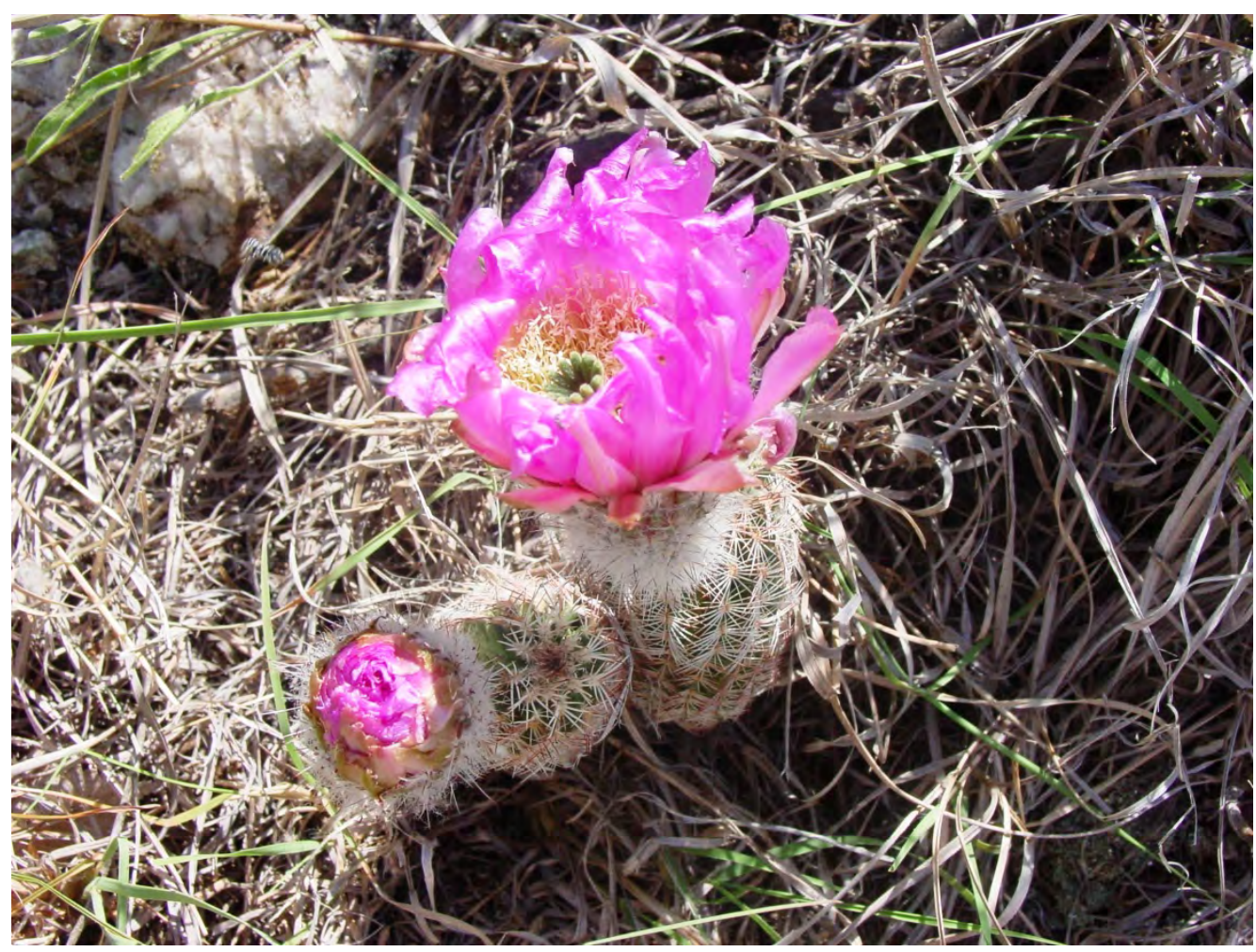

Figure 7 Echinocereus reichenbachii at Alabaster Caverns State Park. Photo by G. Caddell.

(C) Gloria M. Caddell, Kristi D. Rice

Journal compilation (C) 2012 Oklahoma Native Plant Society 